\title{
Early Initial Diagnosis of Gardner Syndrome in a 12-Year-Old Boy
}

\author{
Erich Bryan, Peter Kalina \\ Mayo Clinic, Rochester, USA \\ Email: bryan.erich@mayo.edu
}

Received December 6, 2012; revised January 6, 2013; accepted January 16, 2013

\begin{abstract}
A 12-year-old boy presented to his primary care physician with a painful lump at the angle of the left mandible after being kicked in the jaw during a soccer game. Over the next 2 months the lesion became progressively firmer and fine needle aspiration was attempted. Results were non-diagnostic. Panorex examination revealed a calcified mass contiguous with the left angle of the mandible. Considerations were bony callus secondary to trauma versus a calcified hematoma. A maxillofacial CT showed multiple diffuse craniofacial osseous lesions including the clinically suspected region at the left angle of the mandible. Imaging findings were consistent with multiple craniofacial osteomas and workup for Gardner Syndrome was initiated. Genetic testing was subsequently positive for Familial Adenomatous Polyposis.
\end{abstract}

Keywords: Gardner Syndrome; Osteoma

\section{Introduction}

Gardner Syndrome is a rare variant of Familial Adenomatous Polyposis (FAP) caused by a gene mutation on chromosome 5 [1]. Gardner syndrome is defined as a triad of intestinal polyps and extraintestinal manifestations including osteomas and tumors of the skin and soft tissue. Incidence of Gardner syndrome is between 1 in 4000 and 1 in 12,000 [2]. A hereditary form of colonic polyposis was described by Cripps in 1863. Gardner syndrome was ultimately defined by Gardner in 1950 who later recognized the dental and skeletal abnormalities of the disease [2]. The diagnosis portends significant morbidity. Ninety percent of patients with Gardner Syndrome will have intestinal polyps which, in turn, have a nearly one hundred percent chance of malignant transformation. Prophylactic colectomy including rectal resection is recommended when patients have 30 or more intestinal polyps [2]. Benign osteomas usually precede the development of intestinal polyposis and soft tissue tumors [1]. The following case report attempts to demonstrate the importance of early detection of the disease and how radiologists can aide in early detection with knowledge of the extra-intestinal manifestations and their presentation on imaging.

\section{Case Report}

A 12-year-old boy was kicked in the jaw during a soccer game and presented to his primary care physician and subsequently to an ear nose and throat specialist with
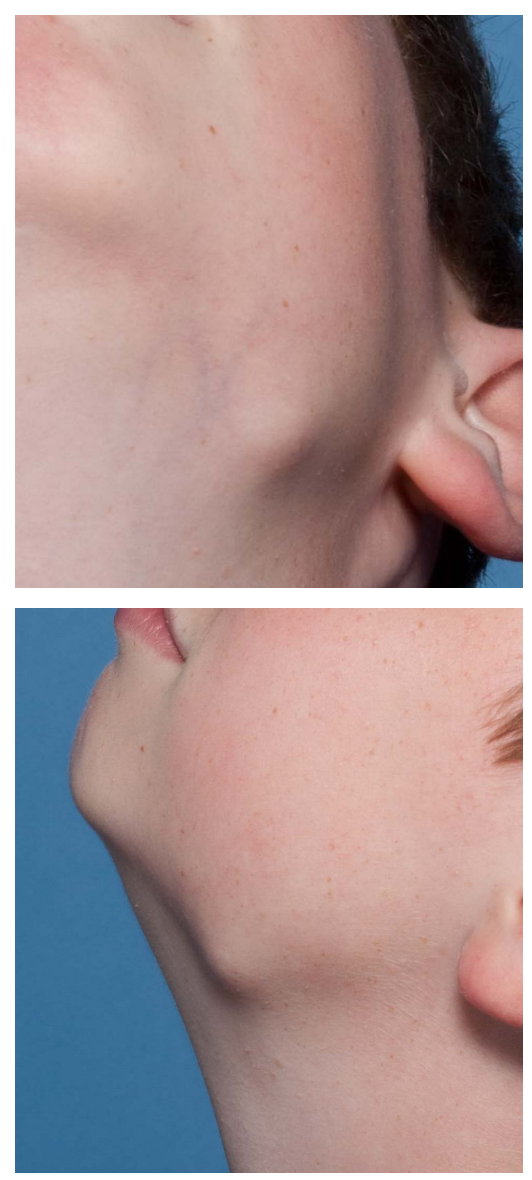

Figure 1. Clinical Photographs of the left mandibular angle lesion. 
complaint of a painless lesion at the angle of the left mandible that appeared to be getting progressively firmer over a two month period (Figure 1). The left mandibular angle lesion and multiple other similar lesions were confirmed on CT to be maxillofacial osteomas. Workup for Gardner Syndrome was initiated and genetic testing was positive.

\section{Discussion}

Gardner Syndrome is a variant of Familial Adenomatous Polyposis. It is an early onset autosomal dominant genetic that can affect both sexes. Intestinal polyps can occur anywhere in the digestive tract, most commonly in the colon. These polyps must be completely removed due to the high rate of malignant degeneration [2]. The average age of diagnosis of intestinal polyps is 22 years though they typically develop at about the time of puberty. Progression to malignancy is typically seen between ages 30 to 60 years [3].

Extra-intestinal manifestations typically precede the intestinal polyps and can include bone lesions (benign osteomas), thyroid carcinoma, connective tissue tumors, and pigmentation of the retina. Soft tissue tumors can include neurofibromas, fibromas, keloids, sebaceous cysts, leiomyomas, lipomas, and desmoid tumors [2]. Osteomas are benign tumors of compact bone that show continuous osseous growth. They are required to make the diagnosis of Gardner Syndrome. These tumors will most frequently be seen involving the teeth, facial bones, and cranium. These lesions are not commonly painful and they are typically removed only for aesthetic reasons or due to limitations in mandibular movement. A typical location for an osteoma is at the angle of the mandible, as in the case presented above [1].

Computed Tomography (CT) is often utilized as the initial imaging modality for the evaluation of potential maxillofacial disease processes. This is true for both soft tissue disease such as such as inflammatory changes as well as osseous abnormalities. CT is preferred due to its increased availability, decreased cost and ability to provide excellent, often superior osseous detail. In our case, a potential focal hematoma or mandibular lesion were suspected due to the trauma history. For both of these indications, CT would be the preferred modality.

This patient's CT scans of the face (including coronal and sagittal reformatted images) demonstrates multiple maxillofacial osteomas including the area of palpable abnormality over the angle of the left mandible where the patient was kicked during his soccer game as well as a large ethmoid lesion (Figures 2 and 3). 3D surface reformatted images confirm and further delineate the extent of the osteomas (Figure 4). The association of multiple maxillofacial osteomas with Gardner syndrome was rec-
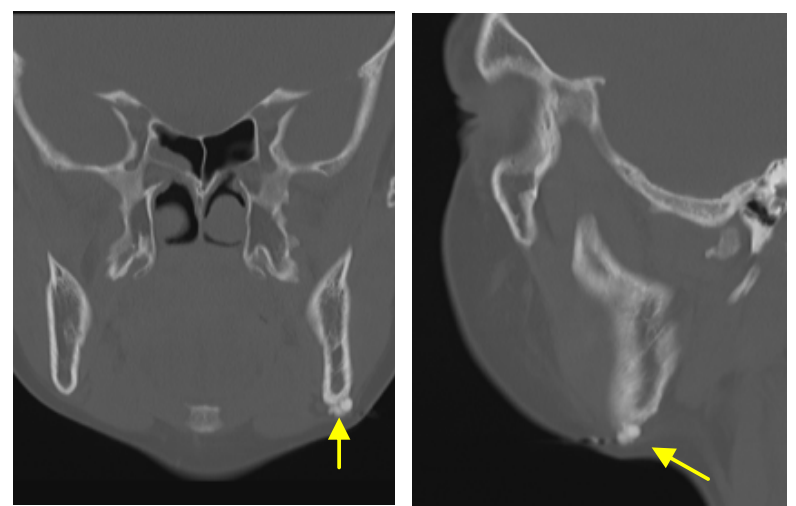

Figure 2. Coronal and Sagittal CT showing left mandibular angle osteoma.
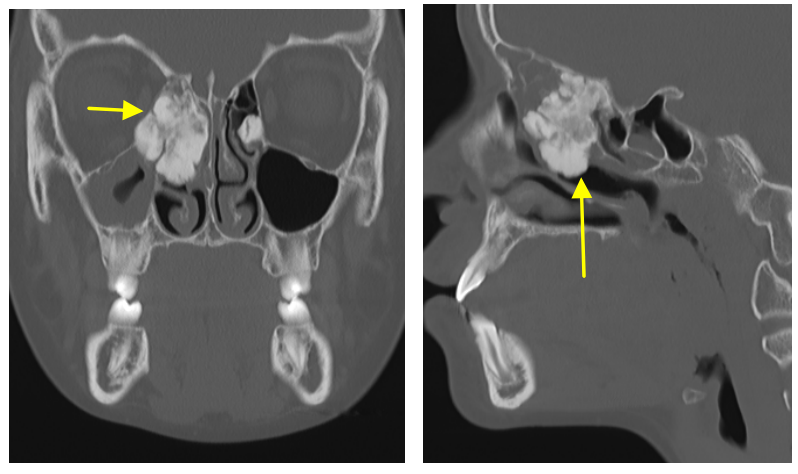

Figure 3. Coronal and Sagittal CT showing mutiple additional craniofacial osteomas including a large ethmoid lesion.
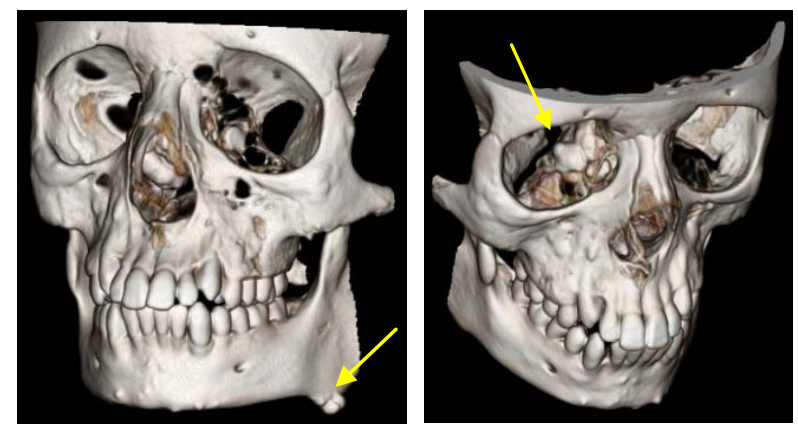

Figure 4. 3D Reformatted CT showing left mandibular and large ethmoid osteomas.

ognized and workup for the disease was initiated. The patient's genetic workup was positive for the variant of Familial Adenomatous Polyposis (FAP). He was then referred to the appropriate services for further screening and management.

In conclusion benign osteomas usually precede the development of intestinal polyposis and soft tissue tumors [1]. Therefore, the radiologist and dentist can diagnose early Gardner Syndrome and help initiate workup with genetics, gastroenterology, oncology, and oral/maxillofacial surgery to hopefully prevent morbity and 
mortality associated with the disease.

\section{REFERENCES}

[1] A. B. Cankaya, M. A.Erdem, S. C. Isler, M. Cifter, V. Olgac, C. Kasapoglu and C. K. Oral, "Oral and Maxillofacial Considerations in Gardner's Syndrome,” International Journal of Medical Science, Vol. 9, No. 2, 2012, pp. 137-141.
[2] U. Bilkay, O. Erdem, C. Ozek, E. Helvaci, K. Kilic, Y. Ertan and T. Gurler, "Benign Osteoma with Gardner Syndrome: Review of the Literature and Report of a Case," Journal of Craniofacial Surgery, Vol. 15, No. 3, 2004, pp. 506-509.

[3] M. Brucoli, M. Giarda and A. Benech, "Gardner Syndrome: Presurgical Planning and Surgical Management of Craniomaxillofacial Osteomas," Journal of Craniofacial Surgery, Vol. 22, No. 3, 2011, pp. 946-948. 